\title{
Deaths, disability-adjusted life years and years of life lost due to elevated systolic blood pressure in Poland: estimates for the Global Burden of Disease Study 2016
}

\author{
Tomasz Miazgowski', Aleksandra Taszarek², Katarzyna Widecka ${ }^{3}$, Bartosz Miazgowski², \\ Katarzyna Homa ${ }^{1}$ \\ ${ }^{1}$ Department of Hypertension and Internal Diseases, Pomeranian Medical University, Szczecin, Poland \\ ${ }^{2}$ Doctoral Study, Pomeranian Medical University, Szczecin, Poland \\ ${ }^{3}$ Department of Cardiology, Pomeranian Medical University, Szczecin, Poland
}

\begin{abstract}
Introduction. High systolic blood pressure (SBP) is a well-known risk factor for major adverse cardiovascular outcomes; however, data regarding disease burden due to high SBP in the Polish population are scarce.

Material and methods. We extracted and analyzed the latest country-, gender-, age- and year-specific estimates from the Global Burden of Disease (GBD) Study 2016 for SBP-related mortality, years of life lost (YLLs), disability-adjusted life years (DALYs), and attributable risk factors in Poland in 2016. In the GBD 2016, the term 'high SBP' refers to SBP of at least $110-115 \mathrm{mmHg}$.

Results. High SBP was attributable to (per 100,000) 106,043.16 deaths (95\% UI [Uncertainty Interval]: 88,207121,849 ) that was $27.22 \%$ of all deaths in Poland in 2016; 1,751,844.69 DALYs (95\% UI: $1,525,188-1,966,25$ ) and 1,497,959.71 YLLs (95\% UI: 1,287,279-1,497,959). In males, DALYs attributable to high SBP were higher by $34 \%$ and YLLs by $23 \%$, while in females death rates were higher by $14 \%$. SBP was highly attributable to ischemic heart disease, stroke, and chronic kidney disease $(63.7 \%, 63,1 \%$, and $59.1 \%$, respectively). In the GBD hierarchy, high SBP was the most common risk factor, followed by smoking, high body mass index (BMI), high total cholesterol levels, alcohol use, and high fasting plasma glucose levels.

Conclusions. In Poland, SBP of at least $110-115 \mathrm{mmHg}$ remains one of the largest risks for loss of good health; greater than smoking, high cholesterol levels, or high BMI. With the population aging globally, the burden due to high SBP is expected to increase further.

Key words: Global Burden of Disease Study, systolic blood pressure, disability-adjusted life years, mortality Arterial Hypertens. 2018, vol. 22, no. 2, pages: 95-103

DOI: 10.5603/AH.a2018.0005
\end{abstract}

\section{Introduction}

Elevated systolic blood pressure (SBP) is a wellknown risk factor for major adverse cardiovascular outcomes, including stroke, myocardial infarction, heart failure, and peripheral artery disease. With the exception of spurious isolated systolic hypertension in young individuals and isolated systolic hypertension in certain endocrine disorders, high SBP typically reflects thickening and stiffness of large arteries, frequently seen in the elderly. The vast majority of

Address for correspondence: Tomasz Miazgowski, Department of Hypertension and Internal Medicine, Pomeranian Medical University, 71-252 Szczecin, tel. +4891 4253550, fax +4891 4253552; e-mail: miazgowski@interia.pl 
current guidelines have recommend an SBP value of $140 \mathrm{mmHg}$ as a cut-off for diagnosis and optimal treatment of arterial hypertension [1, 2]; however, the precise subpopulation(s) and target(s) for the appropriate use of antihypertensive treatment remain under debate. The SPRINT Trial demonstrated significant reductions in mortality in patients with high cardiovascular risk upon receipt of intensive blood pressure reduction therapy and achievement of an average SBP below $120 \mathrm{mmHg}[3,4]$. Similarly, two large meta-analyses showed reductions in cardiovascular-related deaths for a target SBP as low as $120 \mathrm{mmHg}[5,6]$. Other studies have found an SBP of at least of $110 \mathrm{mmHg}$ has to be related to many cardiovascular and renal outcomes [7,8], increased death rates, and higher disability-adjusted life years (DALYs) [9, 10]. DALYs measurement is a common index to quantitate overall burden of disease, expressed as the number of years of life lost (YLLs) and the years lived with disability (YLD) [11]. A number of risk factors are known to contribute to elevated SBP and related comorbidities, including ageing, high cholesterol levels, smoking, hyperglycemia, obesity, high sodium diet, and lead exposure; however, data regarding the burden of elevated SBP attributable to DALYs and YLLs in the Polish population are scarce. Accurate estimates of deaths attributable to elevated SBP, disease burden at the population level, and risk factors for related disability appear necessary to effectively manage this condition in Poland.

In the present report, we extracted and analyzed the latest country-, sex-, age- and year-specific estimates from the Global Burden of Disease (GBD) study - a comprehensive platform for understanding the main drivers of poor health at international, national, and local levels - for SBP-related mortality, YLLs, DALYs, and attributable risk factors in Poland.

\section{Material and methods}

\section{The GBD Study}

The present report is part of the GBD 2016 comparative risk assessment to assess health loss-related to specific risk factors [12-15]. In contrast to cross-sectional or primary studies that analyze individual records to evaluate the number of people with high or normal SBP and related burden, the GBD project provides a descriptive meta-analysis of available study results and other medical records. Hence, the GBD data are projections at the population level rather than direct estimates for a specific sample and should be evaluated considering the availability and reliability of primary data for a given country (or region) and year, uncertainty of the pooled estimates, and the overall modeling and assumptions [10].

\section{Burden related to SBP}

In the GBD study, the term 'elevated SBP' refers to an SBP of at least 110 to $115 \mathrm{mmHg}$. On the basis of analysis from pooled cohort studies, in the GBD 2016 the following diseases were identified as having sufficient evidence to support a relationship with high SBP: ischemic heart disease, ischemic stroke, hemorrhagic stroke, hypertensive heart disease, cardiomyopathy, atrial fibrillation, aortic aneurysm, rheumatic heart disease, peripheral vascular disease, endocarditis, chronic kidney disease, and other cardiovascular diseases. Although the cause of rheumatic heart disease and endocarditis is infection, high SBP has been associated with an increasing risk of death, accelerating adverse heart effects caused by infection or autoimmune response [12].

\section{Computing methods}

SBP-related deaths and DALYs were computed by multiplying a country-, age-, sex-, year-, and cause-specific population-attributable fraction by the estimated deaths or DALYs for the same strata as described elsewhere $[10,13,15]$. The main method used in the fatal outcomes and YLLs estimation was Cause of Death Ensemble modelling (CODEm) [13]. Non-fatal outcomes for a disease were analyzed using the Bayesian meta-regression tool the Disease Model-Meta Regression (DisMod-MR 2.1.) [15]. Death rate and DALYs were determined using the updated GBD 2016 global age-standard population [13].

Using the GBD results standard tool [16], from the GBD 2016 estimates we obtained the estimates for burden of elevated SBP in Poland in 2016. This tool provides access to complete set of age and gender-specific estimates of burden across a wide range of causes. Data were shown as the number of prevalent cases per 100,000 along with $95 \%$ uncertainty interval (UI), which captures uncertainty from sampling, non-sampling error from the study designs or diagnostic methods, model parameter uncertainty, and uncertainty regarding model specification. UIs were generated by taking 1,000 draws from the posterior distribution of each estimate, with upper and lower bounds determined by the $25^{\text {th }}$ and $97.5^{\text {th }}$ values of the draws as described elsewhere [17]. Larger uncertainty intervals can result from limited data availability, small studies, and conflicting data, while smaller uncertainty intervals can result from extensive data availability, large studies, and data that are consistent across sources. 


\section{Risk factors}

SBP-attributable risk factors were estimated based on risk factor prevalence using comparative risk assessment; this tool allows the estimation of the level of mortality and disability related to SBP that can be attributed to past exposure risk [15]. In the GBD study, risk factors were categorized as environmental/ /occupational, behavioral, and metabolic. Attributable burden is defined as the share of the burden of a disease that can be estimated to occur due to exposure to a particular risk factor. Diet high in sodium is defined as consumption of more than 1,000 $\mathrm{mg}$ of sodium per day. Diet high in sugar-sweetened beverages is defined as consumption of any beverage with $\geq 50$ calories of sugar per one-cup serving, including carbonated beverages, sodas, energy drinks, fruit drinks but excluding 100\% fruit and vegetable juices. Diet low in fruits is defined as consumption of less than 3 servings (11 ounces total) of fruits per day (includes fresh, frozen, cooked, canned, or dried fruit but excludes fruit juices and salted or pickled fruits). Diet low in nuts and seeds is defined as consumption of less than 4 servings ( 4 ounces total) of nuts and seeds per week, such as peanut butter. Diet low in whole grains is defined as consumption of less than 2.5 servings ( 4 ounces total) per day of whole grains (bran, germ, and endosperm in their natural proportions) from breakfast cereals, bread, rice, pasta, biscuits, muffins, tortillas, pancakes, and others. High body mass index is defined as above $21.0-23.0 \mathrm{~kg} / \mathrm{m}^{2}$.

The study utilized existing data from the GBD 2016 Study and did not require ethical approval. The GBD 2016 Study complies with the Guidelines for Accurate and Transparent Health Estimates Reporting (GATHER) recommendations [18]. The GBD 2016 estimates are freely available in an interactive data downloading tool on the Global Health Data exchange (GHDx) at: https://vizhub.healthdata.org/ gbd-compare/. The GHDx includes data for causes, risks, cause-risk attribution, aetiologies, and impairments.

\section{Results}

\section{Deaths, DALYs and YLLs attributable to SBP by cause}

The total number of DALYs, YLLs and deaths in 2016 attributable to high SBP of all causes are shown in Table I. In total, the number of deaths attributable to high SBP was 106,043, which accounted for $27.22 \%$ of all deaths in Poland in 2016. The total DALYs attributable to high SBP of all causes were higher by $34 \%$ in males $(1,003,369$ vs. 748,515 in males and females, respectively). Similarly, the total YLLs were greater in males by $23 \%(752,639$ vs. 611,533 in males and females, respectively). In contrast, the death rates were greater in females by $13.6 \%$ (49,651 vs. 56,392 in males and females, respectively).

The percentage risk attribution of SBP to DALYs of cardiovascular outcomes is shown in Table II. As per GBD definition, hypertensive heart disease was fully attributable to high SBP; overall, hypertensive heart disease accounted for less than 1\% of DALYs of all causes. Among other outcomes, SBP was highly attributable to ischemic heart disease, stroke, and chronic kidney disease $(63.7 \%, 63,1 \%$, and $59.1 \%$, respectively), with a slightly stronger attribution to ischemic heart disease- and stroke-DALYs in males, and no clear gender predilection to chronic kidney disease. In males, SBP showed a higher attribution to atrial fibrillation/flutter, aortic aneurysm, endocarditis, peripheral artery disease, and other cardiovascular diseases, with the exception of cardiomyopathy, to which an SBP attribution was higher in females. Among all cardiovascular outcomes, ischemic heart disease had the largest impact on total DALYs (13\%); and similarly, had a large impact on the total YLLs (Table III) and death rate (Table IV). Overall, SBP contributed to YLLs and deaths associated with cardiovascular outcomes in a similar pattern as to DALYs.

As expected, the total DALYs, YLLs, and death rates attributable to high SBP increased with age, as shown in Table $\mathrm{V}$; however, regardless of age,

Table I. Total number of DALYs, YLL and deaths attributable to high systolic blood pressure due to all causes, both sexes combined

\begin{tabular}{|l|c|c|}
\hline Measure & Number & Percent of total \\
\hline Disability-adjusted life years & $1,751,844.69$ & 14.82 \\
& $(1,525,188.94-1,96625.83)$ & $(12.9-16.82)$ \\
\hline Years of life lost & $1,497,959.71$ & 22.09 \\
& $(1,287,279.99-1,497,959.71)$ & $(19.79-24.3)$ \\
\hline Deaths & $106,043.16$ & 27.21 \\
& $(88,207.45-121,849.82)$ & $(23.25-30.03)$ \\
\hline
\end{tabular}


Table II. DALYs attributable to high systolic blood pressure

\begin{tabular}{|l|c|c|c|c|}
\hline \multirow{2}{*}{ Cardiovascular outcomes } & \multicolumn{3}{|c|}{ Risk attribution (\%) } & $\begin{array}{c}\text { \% of total DALYs } \\
\text { (Both sexes) }\end{array}$ \\
\cline { 2 - 5 } & All & Males & Females & $\begin{array}{c}0.67 \\
(0.54-1.09)\end{array}$ \\
\hline Hypertensive heart disease & 100 & 100 & 100 & 12.96 \\
& & & & $(11.56-14.47)$ \\
\hline Ischemic heart disease & 63.68 & 65.47 & 61.09 & 5.53 \\
& $(54.46-72.05)$ & $(57.71-72.63)$ & $(49.26-72.54)$ & $(4.90-6.16)$ \\
\hline Chroke & 63.08 & 66.67 & 59.26 & 1.05 \\
& $(54.76-70.19)$ & $(58.7-3.1)$ & $(50.12-67.57)$ & $(0.98-1.12)$ \\
\hline Atrial fibrillation and flutter & 59.1 & 58.75 & 59.45 & 0.5 \\
& $(51.32-66.32)$ & $(50.81-66.58)$ & $(51.9-66.22)$ & $(0.4-0.6)$ \\
\hline Aortic aneurysm & 46.27 & 48.29 & 45.0 & 0.35 \\
& $(40.24-51.97)$ & $(42.62-53.57)$ & $(38.4-51.18)$ & $(0.3-0.41)$ \\
\hline Endocarditis & 47.59 & 48.79 & 44.32 & 0.093 \\
& $(40.66-53.98)$ & $(41.68-54.98)$ & $(36.77-51.2)$ & $(0.073-0.13)$ \\
\hline Peripheral artery disease & 45.48 & 47.68 & 41.09 & 0.3 \\
& $(37.32-52.61)$ & $(38.79-55.04)$ & $(32.99-48.14)$ & $(0.22-0.4)$ \\
\hline Cardiomyopathy and myocarditis & 34.07 & 35.57 & 32.21 & 1.64 \\
& $(26.57-41.92)$ & $(27.94-42.92)$ & $(29.04-41.0)$ & $(1.28-1.91)$ \\
\hline Other CV and circulatory diseases & 29.05 & 25.15 & 34.52 & 1.37 \\
& $(22.50-36.27)$ & $(19.11-35.34)$ & $(25.88-45.26)$ & $(1.25-1.5)$ \\
\hline
\end{tabular}

Data are percentages (95\% UIs)

Table III. YLLs attributable to high systolic blood pressure

\begin{tabular}{|l|c|c|c|c|}
\hline \multirow{2}{*}{ Cardiovascular outcomes } & \multicolumn{3}{|c|}{ Risk attribution [\%] } & $\begin{array}{c}\text { Percent of total YLL } \\
\text { (Both sexes) }\end{array}$ \\
\cline { 2 - 5 } & All & Males & Females & $\begin{array}{c}0.96 \\
(0.76-1.65)\end{array}$ \\
\hline Hypertensive heart disease & 100 & 100 & 20.97 \\
\hline Ischemic heart disease & 63.71 & 65.49 & 61.06 & $(20.11-21.91)$ \\
\hline Stroke & $(54.45-72.17)$ & $(57.71-72.71)$ & $(48.74-72.58)$ & 7.63 \\
& 63.75 & 67.38 & 59.59 & $(7.13-8.18)$ \\
\hline Chronic kidney disease & $(55.74-70.72)$ & $(59.75-73.95)$ & $(50.36-67.77)$ & 1.08 \\
& 59.78 & 59.09 & 60.7 & $(1.03-1.15)$ \\
\hline Atrial fibrillation and flutter & $(51.85-67.17)$ & $(50.98-66.96)$ & $(52.92-67.66)$ & 0.38 \\
& 42.87 & 43.92 & 42.33 & $(0.29-0.47)$ \\
\hline Aortic aneurysm & $(35.43-49.83)$ & $(36.97-50.32)$ & $(34.44-49.9)$ & 0.61 \\
& 47.59 & 48.79 & 44.32 & $(0.56-0.67)$ \\
\hline Endocarditis & $(40.66-50.63)$ & $(41.68-54.96)$ & $(36.77-51.2)$ & 0.16 \\
& 45.52 & 47.7 & 41.08 & $(0.13-0.23)$ \\
\hline Peripheral artery disease & $(37.34-52.64)$ & $(38.83-55.05)$ & $(33.01-48.13)$ & 0.45 \\
& 33.97 & 35.55 & 31.74 & $(0.31-0.62)$ \\
\hline Cardiomyopathy and myocarditis & $(26.56-41.8)$ & $(27.91-42.95)$ & $(23.55-40.96)$ & 2.62 \\
& $(22.36-36.63)$ & $(18.92-35.71)$ & $(26.02-45.51)$ & $(2.02-2.95)$ \\
\hline Other CV and circulatory diseases & 51.9 & 54.69 & 48.21 & 1.37 \\
& $(47.58-56.59)$ & $(50.18-59.49)$ & $(43.12-52.95)$ & $(1.26-1.5)$ \\
\hline
\end{tabular}


Table VI. Deaths attributable to high systolic blood pressure

\begin{tabular}{|c|c|c|c|c|}
\hline \multirow[t]{2}{*}{ Cardiovascular outcomes } & \multicolumn{3}{|c|}{ Risk attribution (\%) } & \multirow{2}{*}{$\begin{array}{l}\text { Percent of total deaths } \\
\text { (Both sexes) }\end{array}$} \\
\hline & All & Males & Females & \\
\hline Hypertensive heart disease & 100 & 100 & 100 & $\begin{array}{c}1.26 \\
(0.98-2.24)\end{array}$ \\
\hline Ischemic heart disease & $\begin{array}{c}60.13 \\
(46.79-72.72)\end{array}$ & $\begin{array}{c}61.56 \\
(51.26-71.55)\end{array}$ & $\begin{array}{c}58.87 \\
(42.61-73.42)\end{array}$ & $\begin{array}{c}28.72 \\
(27.69-29.91)\end{array}$ \\
\hline Stroke & $\begin{array}{c}57.9 \\
(48.39-67.12)\end{array}$ & $\begin{array}{c}61.8 \\
(53.24-69.32)\end{array}$ & $\begin{array}{c}55.04 \\
(43.86-66.29)\end{array}$ & $\begin{array}{c}9.19 \\
(8.48-9.93)\end{array}$ \\
\hline Chronic kidney disease & $\begin{array}{c}65.07 \\
(57.35-71.77)\end{array}$ & $\begin{array}{c}63.58 \\
(55.89-70.9)\end{array}$ & $\begin{array}{c}66.65 \\
(59.36-73.08)\end{array}$ & $\begin{array}{c}1.21 \\
(1.15-1.29)\end{array}$ \\
\hline Atrial fibrillation and flutter & $\begin{array}{c}39.13 \\
(30.46-47.5)\end{array}$ & $\begin{array}{c}38.98 \\
(30.99-46.77)\end{array}$ & $\begin{array}{c}39.2 \\
(30.28-47.95)\end{array}$ & $\begin{array}{c}0.72 \\
(0.55-0.91)\end{array}$ \\
\hline Aortic aneurysm & $\begin{array}{c}44.36 \\
(36.75-51.52)\end{array}$ & $\begin{array}{c}45.74 \\
(38.25-52.61)\end{array}$ & $\begin{array}{c}41.4 \\
(32.79-49.91)\end{array}$ & $\begin{array}{c}0.58 \\
(0.53-0.63)\end{array}$ \\
\hline Endocarditis & $\begin{array}{c}44.49 \\
(36.23-52.4)\end{array}$ & $\begin{array}{c}46.71 \\
(38.34-53.67)\end{array}$ & $\begin{array}{c}41.26 \\
(32.61-50.93)\end{array}$ & $\begin{array}{c}0.12 \\
(0.099-0.18)\end{array}$ \\
\hline Peripheral artery disease & $\begin{array}{c}31.12 \\
(22.83-40.39)\end{array}$ & $\begin{array}{c}32.98 \\
(25.39-41.13)\end{array}$ & $\begin{array}{c}29.5 \\
(20.12-40.55)\end{array}$ & $\begin{array}{c}0.64 \\
(0.43-0.91)\end{array}$ \\
\hline Cardiomyopathy and myocarditis & $\begin{array}{c}31.43 \\
(22.69-42.52)\end{array}$ & $\begin{array}{c}27.91 \\
(20.56-37.14)\end{array}$ & $\begin{array}{c}34.35 \\
(23.6-50.06)\end{array}$ & $\begin{array}{c}3.27 \\
(2.32-3.78)\end{array}$ \\
\hline Other CV and circulatory diseases & $\begin{array}{c}47.75 \\
(42.56-52.89)\end{array}$ & $\begin{array}{c}50.91 \\
(46.17-55.82)\end{array}$ & $\begin{array}{c}44.82 \\
(38.67-51.03)\end{array}$ & $\begin{array}{c}1.39 \\
(1.27-1.52)\end{array}$ \\
\hline
\end{tabular}

Data are percentages (95\% UIs)

the rates of DALYs were 2-4 times higher in males than in females. Males also had higher rates of YLLs among the age groups, with the exception of the group consisting of individuals over 70 years old, in which the rates were comparable. Overall, the gender differences in YLLs were smaller than in DALYs. The death rates were higher in males, but only in the 15-49 and 50-69 age groups. In the oldest age group (over 70 years old), the rates of both genders were comparable, which was similar for all ages combined.

\section{Risk factors}

At level 1 of the GBD hierarchy for risk factors, among all-cause deaths in Poland in 2016, 491.37 per 100,000 (95\% UI: 441.17-547.45) were attributable to behavioral risks, 428.71 per 100,000 (95\% UI: 383.24-476.73) to metabolic risks, and 99.27 per 100,000 (95\% UI: 86.98-113.9) to environmental and occupational risks. Table VI shows the leading level 3 risk factors for all-cause deaths, DALYs, and YLLs in Poland for both genders combined. High SBP occupied the first position in the GBD 2016 hierarchy, resulting in 27\% of deaths, almost $15 \%$ of DALYs, and $14 \%$ of YLLs. On the second to fifth positions were smoking, high body mass index, high total cholesterol levels, and alcohol use, respectively.
Among all combined deaths for all ages and both genders due to SBP-related cardiovascular outcomes including hypertensive heart disease, ischemic heart disease, stroke, cardiomyopathy, atrial fibrillation, aortic aneurysm, rheumatic heart disease, peripheral vascular disease, endocarditis, chronic kidney disease, and other cardiovascular diseases, the leading attributable risk factors, aside from high SBP, were high fasting plasma glucose levels, smoking, alcohol use, high body mass index, and a high-salt diet, as well as, rather unexpectedly, lead exposure (Table VII).

\section{Discussion}

To the best of our knowledge, this is the first report evaluating the burden associated with an SBP of at least $110-115 \mathrm{mmHg}$ in Poland using the GBD Study 2016. We found that elevated SBP contributed to 106,043 deaths (27\% of all deaths in Poland in 2016), nearly 1.5 million YLLs, and over 1.7 million DALYs. Furthermore, we found that ischemic heart disease and stroke accounted for the majority of loss of good health (deaths, DALYSs, and YLLs) related to an SBP of at least 110-115 mmHg. SBP also showed a high causality of chronic kidney disease, atrial fibrillation and flutter, aortic aneurysm, 
Table V. DALYs and YLLs and death rates per 100,000 attributable to high systolic blood pressure by sex and age

\begin{tabular}{|c|c|c|c|}
\hline Age group & All & Males & Females \\
\hline \multicolumn{4}{|l|}{ DALYs } \\
\hline 5-14 & $\begin{array}{c}0.74 \\
(0.53-0.99)\end{array}$ & $\begin{array}{c}0.73 \\
(0.48-1.03)\end{array}$ & $\begin{array}{c}0.75 \\
(0.55-1.01)\end{array}$ \\
\hline $15-49$ & $\begin{array}{c}656.73 \\
(531.33-782.63)\end{array}$ & $\begin{array}{c}1,020.63 \\
(820.58-1,239.21)\end{array}$ & $\begin{array}{c}282.38 \\
(219.42-350.51)\end{array}$ \\
\hline $50-69$ & $\begin{array}{c}7,650.34 \\
(6,657.55-8,547.11)\end{array}$ & $\begin{array}{c}11,451.31 \\
(9,724.35-13,142.52)\end{array}$ & $\begin{array}{c}4,239.92 \\
(3,586.4-4,866.86)\end{array}$ \\
\hline $70+$ & $\begin{array}{c}21,266.68 \\
(17,696.11-24,482.38)\end{array}$ & $\begin{array}{c}24,568.3 \\
(20,363.76-28,959.65)\end{array}$ & $\begin{array}{c}19,416.6 \\
(15,687.05-22.773 .36)\end{array}$ \\
\hline All ages & $\begin{array}{c}4,533.65 \\
(3,946.99-5.088 .43)\end{array}$ & $\begin{array}{c}5,368.31 \\
(4,619.42-6,131.77)\end{array}$ & $\begin{array}{c}3,751.73 \\
(3,138.11-4,297.36)\end{array}$ \\
\hline \multicolumn{4}{|l|}{ YLLs } \\
\hline 5-14 & $\begin{array}{c}0.03 \\
(0.015-0.053)\end{array}$ & $\begin{array}{c}0.033 \\
(0.016-0.059)\end{array}$ & $\begin{array}{c}0.023 \\
(0.013-0.047)\end{array}$ \\
\hline $15-49$ & $\begin{array}{c}108.54 \\
(72.59-148.57)\end{array}$ & $\begin{array}{c}131.86 \\
(89.11-180.79)\end{array}$ & $\begin{array}{c}84.56 \\
(56.63-117.7)\end{array}$ \\
\hline $50-69$ & $\begin{array}{c}1,114.85 \\
(792.18-1,469.36)\end{array}$ & $\begin{array}{c}1,282.66 \\
(915.57-1,704.91)\end{array}$ & $\begin{array}{c}964.28 \\
(678.68-1,256.22)\end{array}$ \\
\hline $70+$ & $\begin{array}{c}3,004.06 \\
(2,184.14-3,929.36)\end{array}$ & $\begin{array}{c}2,955.81 \\
(2,160.03-3,855.57)\end{array}$ & $\begin{array}{c}3,031.1 \\
(2,197.25-3,973.4)\end{array}$ \\
\hline All ages & $\begin{array}{c}657.13 \\
(473.51-855.69)\end{array}$ & $\begin{array}{c}625.68 \\
(448.2-817.34)\end{array}$ & $\begin{array}{c}868.58 \\
(494.04-888.85)\end{array}$ \\
\hline \multicolumn{4}{|l|}{ Deaths } \\
\hline $5-14$ & $\begin{array}{c}0.0092 \\
(0.0066-0.013)\end{array}$ & $\begin{array}{c}0.0091 \\
(0.0059-0.013)\end{array}$ & $\begin{array}{c}0.0094 \\
(0.0069-0.013)\end{array}$ \\
\hline $15-49$ & $\begin{array}{c}12.57 \\
(10.15-15.26)\end{array}$ & $\begin{array}{c}20.34 \\
(15.99-25.09)\end{array}$ & $\begin{array}{c}4.59 \\
(3.55-5.77)\end{array}$ \\
\hline $50-69$ & $\begin{array}{c}250.57 \\
(218.05-283.16)\end{array}$ & $\begin{array}{c}385.73 \\
(328.16-448.93)\end{array}$ & $\begin{array}{c}129.3 \\
(108.91-150.37)\end{array}$ \\
\hline $70+$ & $\begin{array}{c}1,948.68 \\
(1,566.87-2,335.33)\end{array}$ & $\begin{array}{c}2,036.69 \\
(1,630.38-2,437.7)\end{array}$ & $\begin{array}{c}1,930.26 \\
(1,505.62-2,329.83)\end{array}$ \\
\hline All ages & $\begin{array}{c}274.43 \\
(228.27-315.33)\end{array}$ & $\begin{array}{c}265.65 \\
(223.3-311.0)\end{array}$ & $\begin{array}{c}282.65 \\
(226.3-335.42)\end{array}$ \\
\hline
\end{tabular}

endocarditis, peripheral artery disease, cardiomyopathy, and other cardiovascular diseases.

Although the total number of DALYs, YLLs and deaths in both genders was age-dependent in the present study, the vast majority of the rates were higher in males than in females. This finding may suggest that females have better health-seeking behavior, are more sensitive to information regarding healthy life styles, and have better access to all aspects of primary prevention of cardiovascular disease. Similar suggestions also arose from estimates for the GBD Study 2013 regarding ischemic heart disease [19] and stroke [20].

In Poland in 2016, an SBP of at least 110-115 $\mathrm{mmHg}$ was ranked number 1 in terms of deaths attributable to risk factors ( $27 \%$ of all deaths), followed by smoking, high cholesterol levels, and high body mass index. An unhealthy diet and pollution were also highly ranked. Other common risk factors in Poland included high fasting blood glucose levels, alcohol use, a high-salt diet, lead exposure, a diet low in fruits, fiber, omega- 3 and vegetables, exposure to occupational carcinogens, and low physical activity. These findings demonstrate that there is huge potential for risk prevention to reduce the deaths, DALYs, and YLLs attributable to high SBP, since all the risk factors included in the present report are modifiable.

Although the GBD 2016 approach was based on the most comprehensive data from 1990 to 2016 and utilized the latest advances in modelling techniques and strategy, it had some limitations, as described elsewhere [12-14], which also apply to the present report. Firstly, an SBP of 110-115 $\mathrm{mmHg}$ was used 


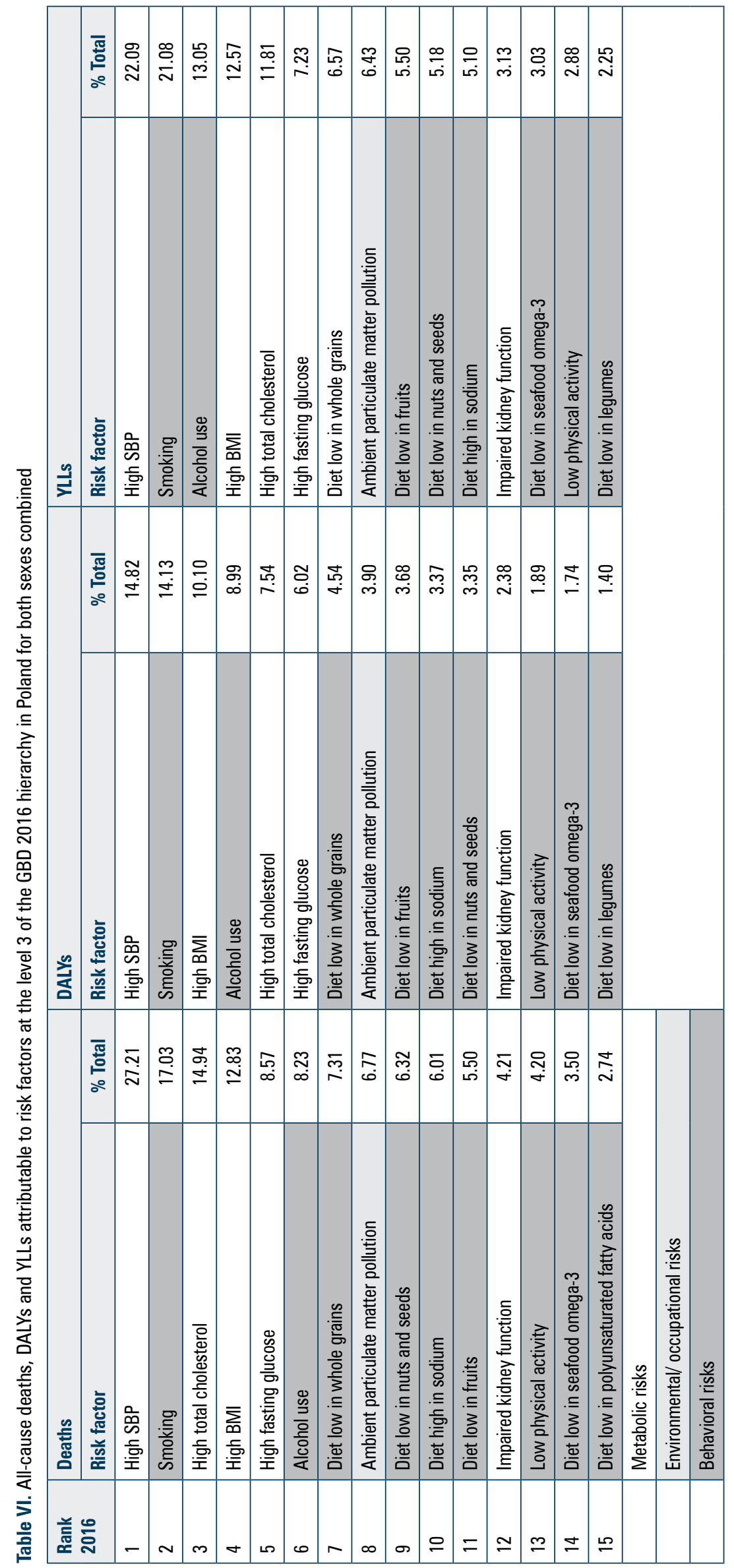

www.ah.viamedica.pl 


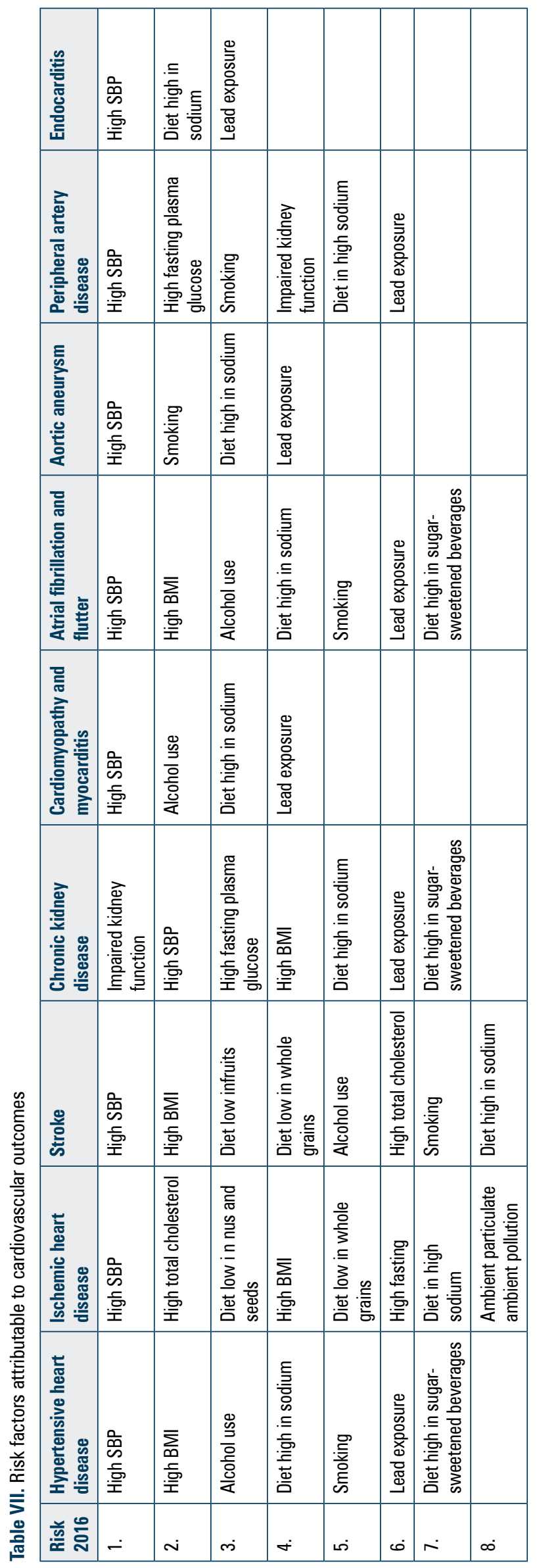

in the GBD Study 2016, corresponding to optimal blood pressure, i.e. below the threshold for diagnosis of hypertension and use of antihypertensive therapies [1]. The SPRINT trial $[3,21]$ and recent meta-analysis [5] have demonstrated a significant reduction in mortality among individuals with high cardiovascular risk upon receipt of intensive blood pressure lowering therapy and achievement of an average SBP below $120 \mathrm{mmHg}$; however, other studies have yielded contradicting results [22, 23]. The present results do not help to resolve the important debate about appropriate clinical use and targets for antihypertensive treatments, they confirm only the model assumption that an SBP of $110-115 \mathrm{mmHg}$ is a modifiable risk factor for mortality and loss of good health, but cannot determine the optimal SBP for the Polish population. Secondly, in the GBD Study 2016 high diastolic blood pressure was not included in the analyses. Thirdly, GBD analyses of SBP in Poland, as in other countries, could be affected by the allocation of garbage codes as well as incorrect mapping to the ICD-10 codes, for example heart failure instead of ischemic heart disease.

Since both the projected number and prevalence rate of an SBP of at least $110-115 \mathrm{mmHg}$ are likely to continue to increase globally [10], the present study has some potential implications for the Polish population. Policies and strategies should be guided by the comparative importance of health loss from exposure to an SBP of at least $110-115 \mathrm{mmHg}$ and other major risk factors for cardiovascular outcomes, aiming to mitigate disease burden through the optimal control of high SBP and a combination of behavioral, lifestyle, and drug treatment strategies.

In conclusion, the present study demonstrates that an SBP of at least 110-115 $\mathrm{mmHg}$ remains one of the largest risks for loss of good health in Poles; greater than smoking, high cholesterol levels, and high body mass index. With the population aging and the fact that SBP levels increase with age globally [10], the number of individuals with hypertension and related adverse health outcomes is expected to increase in Poland.

\section{Funding sources}

The GBD Study is funded by the Bill \& Melinda Gates Foundation. This work was supported by the scientific grant obtained from Pomeranian Medical University in Szczecin (Grant for Young Researchers No. MB-315-227-17).

\section{References}

1. Polish Society of Hypertension. Guidelines for the management of hypertension. Arterial Hypertens. 2015; 19: 53-83. 
2. Cuspidi C, Tadic M, Grassi G, et al. Treatment of hypertension: The ESH/ESC guidelines recommendations. Pharmacol Res. 2018; 128: 315-321, doi: 10.1016/j.phrs.2017.10.003, indexed in Pubmed: 29080798.

3. Wright JT, Williamson JD, Whelton PK, et al. SPRINT Research Group. A Randomized Trial of Intensive versus Standard Blood-Pressure Control. N Engl J Med. 2015; 373(22): 2103-2116, doi: 10.1056/NEJMoa1511939, indexed in Pubmed: 26551272.

4. Williamson JD, Supiano MA, Applegate WB, et al. SPRINT Research Group. Intensive vs Standard Blood Pressure Control and Cardiovascular Disease Outcomes in Adults Aged $\geq 75$ Years: A Randomized Clinical Trial. JAMA. 2016; 315(24): 2673-2682, doi: 10.1001/jama.2016.7050, indexed in Pubmed: 27195814.

5. Ettehad D, Emdin CA, Kiran A, et al. Blood pressure lowering for prevention of cardiovascular disease and death: a systematic review and meta-analysis. Lancet. 2016; 387(10022): 957-967, doi: 10.1016/S0140-6736(15)01225-8, indexed in Pubmed: 26724178 .

6. Law MR, Morris JK, Wald NJ. Use of blood pressure lowering drugs in the prevention of cardiovascular disease: meta-analysis of 147 randomised trials in the context of expectations from prospective epidemiological studies. BMJ. 2009; 338: b1665, doi: 10.1136/ bmj.b1665, indexed in Pubmed: 19454737.

7. Wright JT, et al. Jr, Bakris G, Greene T, African American Study of Kidney Disease and Hypertension Study Group. Effect of blood pressure lowering and antihypertensive drug class on progression of hypertensive kidney disease. JAMA. 2002; 288(19): 2421-2431.

8. Van Gaal LF, Mertens IL, De Block CE. Mechanisms linking obesity with cardiovascular disease. Nature. 2006; 444(7121): 875-880, doi: 10.1038/nature05487, indexed in Pubmed: 17167476.

9. Krishnamurthi RV, Moran AE, Feigin VL, et al. GBD 2013 Stroke Panel Experts Group. Stroke Prevalence, Mortality and Disability-Adjusted Life Years in Adults Aged 20-64 Years in 1990-2013: Data from the Global Burden of Disease 2013 Study. Neuroepidemiology. 2015; 45(3): 190-202, doi: 10.1159/000441098, indexed in Pubmed: 26505983.

10. Forouzanfar MH, Liu P, Roth GA, et al. Global Burden of Hypertension and Systolic Blood Pressure of at Least 110 to 115 mmHg, 1990-2015. JAMA. 2017;317(2): 165-182, doi: 10.1001/ jama.2016.19043, indexed in Pubmed: 28097354.

11. Polinder S, Haagsma JA, Stein C, et al. Systematic review of general burden of disease studies using disability-adjusted life years. Popul Health Metr. 2012; 10(1): 21, doi: 10.1186/1478-7954-10-21, indexed in Pubmed: 23113929.

12. GBD 2016 Disease and Injury Incidence and Prevalence Collaborators. Global, regional, and national incidence, prevalence, and years lived with disability for 328 diseases and injuries for 195 countries, 1990-2016: a systematic analysis for the Global Burden of Disease Study 2016. Lancet. 2017; 390(10100): 1211-1259, doi: 10.1016/ S0140-6736(17)32154-2, indexed in Pubmed: 28919117.

13. GBD 2016 Causes of Death Collaborators. Global, regional, and national age-sex specific mortality for 264 causes of death, 19802016: a systematic analysis for the Global Burden of Disease Study
2016. Lancet. 2017; 390(10100): 1151-1210, doi: 10.1016/S01406736(17)32152-9, indexed in Pubmed: 28919116.

14. GBD 2016 DALYs and HALE Collaborators. Global, regional, and national disability-adjusted life-years (DALYs) for 333 diseases and injuries and healthy life expectancy (HALE) for 195 countries and territories, 1990-2016: a systematic analysis for the Global Burden of Disease Study 2016. Lancet. 2017; 390(10100): 1260-1344, doi: 10.1016/S0140-6736(17)32130-X, indexed in Pubmed: 28919118.

15. GBD 2016 Risk Factors Collaborators. Global, regional, and national comparative risk assessment of 84 behavioural, environmental and occupational, and metabolic risks or clusters of risks, 1990-2016: a systematic analysis for the Global Burden of Disease Study 2016. Lancet. 2017; 390(10100): 1345-1422, doi: 10.1016/S01406736(17)32366-8, indexed in Pubmed: 28919119.

16. Global Burden of Disease Collaborative Network. Global Burden of Disease Study 2016 (GBD 2016) Results. Seattle, United States: Institute for Health Metrics and Evaluation (IHME),2017. http:// ghdx.healthdata.org/gbd-results-tool.

17. Institute for Health Metrics and Evaluation (IHME). Rethinking development and health: Findings from the Global Burden of Disease Study. Seattle, WA, USA: IHME; 2016.

18. Stevens GA, Alkema L, Black RE, et al. Grupo de trabalho GATHER, GATHER Working Group, (The GATHER Working Group), GATHER Working Group. Guidelines for Accurate and Transparent Health Estimates Reporting: the GATHER statement. PLoS Med. 2016; 13(6): e1002056-e23, doi: 10.1371/journal.pmed.1002056, indexed in Pubmed: 27351744.

19. Xie XX, Zhou WM, Lin F, et al. Ischemic heart disease deaths, disability-adjusted life years and risk factors in Fujian, China during 1990-2013: Data from the Global Burden of Disease Study 2013. Int J Cardiol. 2016; 214: 265-269, doi: 10.1016/j.ijcard.2016.03.236, indexed in Pubmed: 27077547.

20. Barker-Collo S, Bennett DA, Krishnamurthi RV, et al. GBD 2013 Writing Group, GBD 2013 Stroke Panel Experts Group. Sex Differences in Stroke Incidence, Prevalence, Mortality and Disability-Adjusted Life Years: Results from the Global Burden of Disease Study 2013. Neuroepidemiology. 2015; 45(3): 203-214, doi: 10.1159/000441103, indexed in Pubmed: 26505984.

21. Still CH, Rodriguez CJ, Wright JT, et al. SPRINT Writing Group. Clinical Outcomes by Race and Ethnicity in the Systolic Blood Pressure Intervention Trial (SPRINT): A Randomized Clinical Trial. Am J Hypertens. 2017; 31(1): 97-107, doi: 10.1093/ajh/hpx138, indexed in Pubmed: 28985268.

22. Di Nora C, Cioffi G, Iorio A, et al. Systolic blood pressure target in systemic arterial hypertension: Is lower ever better? Results from a community-based Caucasian cohort. Eur J Intern Med. 2018; 48: 57-63, doi: 10.1016/j.ejim.2017.08.029, indexed in Pubmed: 28893522.

23. Xie X, Xu J, Gu H, et al. The J-curve Association between Systolic Blood Pressure and Clinical Outcomes in Ischemic Stroke or TIA: The BOSS Study. Sci Rep. 2017; 7(1): 14023, doi: 10.1038/s41598017-10887-w, indexed in Pubmed: 29070878. 